\title{
Mirada general al currículo ecuatoriano y su aplicación
}

Liliana Emperatriz Ordoñez T. ${ }^{5}$

liliana.ordonez@feyalegria.org.ec

Artículo recibido en octubre de 2018 y aprobado en noviembre de 2018.

\section{Resumen}

Es importante hacer memoria y reflexionar los aspectos que contiene el currículo nacional en beneficio de una mejor calidad educativa. En la actualidad existen algunos elementos que con su aplicación podríamos lograr aprendizajes significativos, sin embargo, existen centros educativos que mantienen una educación tradicional y no generan innovaciones educativas, y de todo ello, se desprende varios cuestionamientos al currículo nacional y su aplicación. Además, probablemente deben existir muchas razones para que hoy por hoy existan muchos problemas sociales, políticos y económicos, sin embargo, la educación que es una base para una sociedad más solidaria y justa no está siendo ese elemento de transformación. Por ello, la generación de alternativas de solución y la toma de decisiones es urgente, y se debe empezar a través de espacios de reflexión, con la finalidad de dar soluciones a la falta de prácticas educativas transformadoras que den respuesta a las problemáticas actuales en los centros educativos.

Palabras claves: currículo, calidad, cuestionamientos, estructura y aplicación.

\section{Abstract}

It is important to remember and reflect the aspects that contains

5 Máster Universitario en Liderazgo y Dirección de Centros Educativos por la Universidad Internacional de la Rioja (UNIR) España. Licenciada en Ciencias de la Educación mención Psicología Educativa (UCE). Estudios en Innovación en el Aula, Prevención de Abuso Sexual de niños, niñas y adolescentes, Gestión de talento humano, otros. Experiencia en Asesoría Académica, talleres en Técnicas de estudio, Autoestima, Comunicación, otros. Instructora de Laboratorio de la carrera de Psicología Educativa en Universidad Central del Ecuador. Docente y psicóloga de centros educativos. Actualmente Coordinadora Nacional de Educación General Básica Fe y Alegría Ecuador. 
the national curriculum to the benefit of a better quality of education. At present there are some elements that with your application we could achieve meaningful learning, however, there are educational centers that maintain a traditional education and do not generate educational innovations, and all of this has seen several challenges to the national curriculum and its implementation. In addition, probably there must be many reasons for that today there are many social, political and economic problems, however, education that is a basis for a society with greater solidarity and fair is not being that element of transformation. Therefore, the generation of alternatives of solution and decision-making is urgent, and a start must be made through reflection spaces, with the purpose of giving solutions to the lack of educational practices for transformation that will respond to the current problems in the educational centers.

Key Words: study program, quality, questioning, structure and application.

\section{Introducción}

Sabiendo que el currículo es una construcción histórica cultural, no podemos dejar pasar el tiempo sin hacer memoria y revisar nuestra situación actual con relación a su estructura y su aplicación. Además, es importante tomar en cuenta que es responsabilidad de los gobiernos y de las sociedades hacer todos los esfuerzos para que las diferencias individuales, socioeconómicas, étnicas, de género y de lengua, no se transformen en desigualdad de oportunidades o en cualquier forma de discriminación, ya que, la generación de un currículo sin las personas involucradas en el proceso educativo, es como nadar contra corriente, jamás lograremos avanzar ni generar transformación de nuestra realidad. Cuando se quiere un mundo mejor, es necesario cambiar nuestra forma de pensar y mantener una mente abierta para comprender que la educación no se limita al hecho de repetir las tablas de multiplicar o las sílabas, ya que, implica una comprensión crítica de la realidad social, política y económica en la que está la persona. Tampoco es suficiente con establecer leyes o hacer instructivos, es pensar si nuestros educadores/ as están realmente preparados para estos cambios y cómo podemos desde el cuidado brindarles las herramientas necesarias, sabiendo que cada uno somos producto de una educación tradicional y, por ende, hay que reestructurar nuestros aprendizajes. 
Por tal razón, en este espacio se comparte algunas ideas generales establecidas en el currículo, la Ley Orgánica Intercultural Bilingüe y su reglamento, la cual aporta la mejora de la calidad educativa, con la finalidad de analizar la coherencia entre lo escrito y lo aplicado en sus centros educativos y, por ende, realizar los ajustes necesarios. Así como, la recogida de experiencias y observaciones con relación a la aplicación de las ideas establecidas en el currículo y finalmente algunas posibles alternativas generales.

\section{Aspectos del currículo}

Conocemos que el currículo es una construcción histórica-cultural, la cual ha pasado por diversas acepciones, pese a ello, el currículo debe enfrentarse a la complejidad, la incertidumbre, la contradicción y la ambigüedad como elementos constituyentes Figueroa (como se citó en Rojas, 2012) y por ende ser contextualizado a las realidades de cada país. Además, como indica Martínez: (como se citó en Rojas, 2012)

El campo del currículum requiere ser visto como una totalidad, esto es, como una articulación compleja de procesos, hechos educativos y relaciones entre diferentes sujetos, los que a su vez requieren ser analizados en términos de sus relaciones con otros procesos y con otros sujetos sociales; $y$, al mismo tiempo, como una estructura organizada en cuanto estructura de relaciones significativas que comprende y a su vez explica cada hecho o conjunto de hechos educativos y es, al mismo tiempo, un modo de organización de las prácticas educativas que articula el proyecto educativo con su especificidad disciplinaria (2009, p. 141).

En este sentido, en el país se dieron varios cambios con relación a la educación y a la transformación curricular, por ello, en la LOEI se establecieron derechos y obligaciones en torno a la educación, del cual, uno de los derechos determinados en el Art. 2 es, garantizar el derecho de las personas a una educación de calidad y calidez, pertinente, adecuada, contextualizada, actualizada y articulada en todo el proceso educativo. Así mismo, las obligaciones adicionales descritas con relación al currículo en el literal "I" es, "incluir en los currículos de estudio, de 
manera progresiva, la enseñanza de, al menos, un idioma ancestral; el estudio sistemático de las realidades y las historias nacionales no oficiales, así como de los saberes locales". En este sentido, si nos detenemos en estas afirmaciones, podemos evidenciar que, por parte de la Ley, existe interés y obligación en hacer que, las prácticas educativas de las instituciones educativas brinden una educación contextualizada. Además, otra obligación importante que se establece en la ley en el literal " $g$ " es que, el currículo se debe complementar de acuerdo a las especificidades culturales y peculiaridades propias de las diversas instituciones educativas que son parte del Sistema Nacional de Educación.

Estos avances importantes se fortalecen en la aplicación de los currículos nacionales, por ello, es obligatoria en todas las instituciones educativas del país según el Art. 9 del Reglamento General de la LOEl ${ }^{6}$. Con la finalidad que esto sea posible el Ministerio de Educación realizó una actualización al currículo del 2010.

El avance de la ciencia, los intereses y necesidades del país y el requerimiento de proporcionar a los docentes un currículo más abierto y flexible, que se pudiera adaptar de mejor manera a los estudiantes se realizó una revisión del currículo nacional ecuatoriano, que se llevó a cabo con la participación de distintos actores involucrados en educación, docentes de los diferentes niveles educativos, padres de familia, estudiantes, y representantes del sector productivo del país, así como con la asesoría extranjera (Currículo, 2016, p. 7).

A partir de esa revisión se determina un currículo "sólido, bien fundamentado, técnico, coherente y ajustado a las necesidades de aprendizaje de la sociedad de referencia" (MINEDUC, 2016), del cual se desprende las siguientes funciones:

- Informar a los docentes sobre qué se quiere conseguir y proporcionarles pautas de acción y orientaciones sobre cómo conseguirlo.

6 Ley Orgánica de Educación Intercultural. 
- Construir un referente para la rendición de cuentas del sistema educativo y para las evaluaciones de la calidad del sistema.

Además, nuestro currículo actual (tanto de inicial, educación básica, bachillerato como intercultural bilingüe) brinda la flexibilidad y apertura, lo cual genera una mayor autonomía a las instituciones educativas y a los docentes en la planificación y diseño de la acción educativa, abriendo un espacio de responsabilidad compartida en el desarrollo del currículo (MINEDUC, 2016).

Con ello, se propone un perfil de salida del bachillerato articulado con todos los subniveles, los cuales son definidos a partir de 3 valores fundamentales: lajusticia, la innovación y la solidaridad. Para la ejecución de este perfil se desarrolla, elementos, principios, ejes, orientaciones metodológicas, indicadores, entre otros. Así mismo, otro ajuste es el de la carga horaria por materia, pues ahora podemos modificar las áreas instrumentales, se pueden insertar nuevas materias siempre que éstas tengan una razón de ser, y una finalidad bien sustentada.

\section{Algunas cuestiones pedagógicas}

"Una propuesta teórico-práctica de las experiencias de aprendizaje básicas, diversificadas e innovadoras, que la escuela en colaboración con su entorno debe ofrecer al alumnado para que consiga el máximo desarrollo de capacidades y dominio de competencias, que le permitan integrarse satisfactoriamente en su contexto logrando una sociedad democrática y equitativa" (Casanova, 2006, p. 89)

En el proceso de enseñanza-aprendizaje tanto el componente teórico como práctico basado en las experiencias y en las realidades de cada contexto permitirá un aprendizaje más significativo en los estudiantes, que como nos menciona Casanova el desarrollo máximo de sus capacidades les permitirá integrarse satisfactoriamente a su contexto, no obstante, también es necesario el desarrollo integral (humano, espiritual, reflexivo y crítico), que le permita generar transformación de las realidades de su contexto.

En Fe y Alegría el proceso de enseñanza-aprendizaje está compuesto por 3 componentes: Planificación, Promoción de los 
aprendizajes y Evaluación, por ello vamos a recordar la estructura pedagógica del currículo diferenciando estos 3 componentes.

\section{Planificación}

La planificación es la base que conduce a la concreción de un currículo y sobre todo a las respuestas de las problemáticas y necesidades de cada estudiante. El instructivo de planificación que sugiere el Ministerio de Educación, considera a la planificación como el instrumento que permite organizar y conducir los procesos de enseñanza y aprendizaje necesarios para la consecución de los objetivos educativos.

Además, lleva a reflexionar y tomar decisiones oportunas, pertinentes, tener claro qué necesidades de aprendizaje poseen los estudiantes, qué se debe llevar al aula y cómo se puede organizar las estrategias metodológicas, proyectos y procesos para que el aprendizaje sea adquirido por todos, y de esta manera dar atención a la diversidad de estudiantes" AFCEGBR (como se citó en MINEDUC, 2017).

En el componente de planificación el currículo nacional estipula que,

Si la Autoridad Nacional es responsable de diseñar el currículo obligatorio, las unidades educativas deben acercar este diseño a la realidad de sus contextos a través del Proyecto Educativo Institucional y su correspondiente Proyecto Curricular Institucional y los docentes han de negociar los contenidos en el espacio del aula atendiendo a los intereses y necesidades de sus estudiantes. (MINEDUC, 2016)

En este sentido, en el currículo se establece que los formatos de las planificaciones curriculares son referenciales, por lo que, pueden ser modificados en cada centro educativo atendiendo sus particularidades y contexto, siempre y cuando se mantengan los elementos esenciales (fines, objetivos, contenidos, metodología, recursos y evaluación).

La construcción de planificaciones según el instructivo de planificación del MINEDUC es de manera conjunta entre las autoridades y docentes. 


\section{Promoción de los aprendizajes}

Para la promoción de los aprendizajes el currículo establece objetivos que constituyen una secuencia hacia el logro del perfil de salida:

- Contenidos expresados en Destrezas con Criterio de Desempeño

- Orientaciones metodológicas

En el currículo actual menciona metodologías propias del socio constructivismo para todas las áreas, así como, las activas, participativas, globalizadoras que fomenten el pensamiento racional y crítico y la interdisciplinariedad; trabajo individual, cooperativo e investigativo; respeto por la individualidad y diversidad (ritmos-estilos de aprendizaje) y la utilización de las TICs. También, establece el trabajo por proyectos como el aprendizaje basado en proyectos (ABP) y el juego como estrategia de aprendizaje.

\section{Evaluación}

Con relación a la evaluación estudiantil el MINEDUC lo concibe como "un proceso continuo de observación, valoración y registro de información que evidencia el logro de objetivos de aprendizaje de los estudiantes, mediante sistemas de retroalimentación que están dirigidos a mejorar la metodología de enseñanza y los resultados de aprendizaje", según lo determina el artículo 184 del Reglamento General a la LOEI. Además, en el Art. 16 menciona que el Instituto Nacional de Evaluación Educativa es una instancia encargada de la evaluación integral, interna y externa, del Sistema Nacional de Educación, y en el Art. 19 menciona la evaluación del Aprendizaje, que incluye el rendimiento académico de estudiantes y la aplicación del currículo en instituciones educativas como uno de los componentes que son evaluados por el Sistema.

Además, en el instructivo de evaluación estudiantil establecido por el MINEDUC, estipula que:

Es importante tener claro que la evaluación no es sinónimo de calificación, en tal sentido debe responder al desarrollo integral de los estudiantes, considerando sus logros individuales y los factores que pueden incidir 
en sus aprendizajes. Lo esencial de la evaluación es proveer de retroalimentación al estudiante para que pueda mejorar y lograr los mínimos establecidos que aseguren su aprendizaje, así como para el cumplimiento de los estándares nacionales. La evaluación tiene como propósito principal que el docente oriente al estudiante de manera oportuna, pertinente, precisa y detallada, para ayudarlo a lograr sus objetivos de aprendizaje; la evaluación debe inducir al docente a un proceso de análisis y reflexión valorativa de su trabajo como facilitador de los procesos de aprendizaje, con el objeto de mejorar la efectividad de su gestión. (MINEDUC, 2017)

Para dar orientaciones a este proceso de evaluación se generan Criterios e indicadores de evaluación, y determinan que cada institución educativa proponga su política de evaluación del aprendizaje en su Planificación Curricular Institucional (PCI), considerando el contexto en que se ubica la institución y la normativa vigente presente en su instructivo.

¿Se aplica el currículo actual?: Cuestionamientos

En el apartado anterior hemos recordado aspectos generales sobre el currículo nacional en el cual visualizamos aspectos de mejora al sistema educativo tradicional, hoy por hoy, a través de estos cambios se pretende que la calidad educativa mejore y que nuestros estudiantes tengan un aprendizaje significativo que responda a sus necesidades y que les permita transformar su realidad. Todo lo escrito es un ideal, mientras tanto, cada una de estas afirmaciones no se apliquen en los centros educativos fiscales, fiscomisionales y particulares. De allí, surge el primer cuestionamiento ¿En los centros educativos se aplican estas particularidades positivas que buscan una mejora en la calidad educativa?

Luego de la experiencia de trabajo, de las entrevistas a padres/ madres de familia, diálogos con educadores/as y observaciones presenciales del desenvolvimiento de las instituciones educativas, me permito afirmar que el currículo tiene dificultades de aplicación, así como algunos aspectos a repensar, estas afirmaciones son basadas por lo siguiente: 
- Mientras el currículo menciona que es flexible y abierto, en algunos casos los/las educadores/as temen realizar alguna innovación, ya que, en el momento de auditoría, éstas exigen que los documentos estén tal cual lo prescribe el MINEDUC.

- Existe una inconsistencia entre el instructivo de planificaciones en donde establece que la construcción del PEI es colectivo con la participación de autoridades y docentes, mientras en la LOEI se establece que el estado debe "Garantizar la participación activa de estudiantes, familias y docentes en los procesos educativos" por ello si bien menciona que la construcción es colectiva, por qué no se visibiliza al resto de actores educativos en su construcción.

- Existe deficiencia en la organización de los aspectos administrativos que deben presentar las instituciones, por tal razón, en muchos de los centros educativos quien realiza las planificaciones $(\mathrm{PEI}, \mathrm{PCl}$, PCA) es el director o el equipo Directivo, no existe la participación de la comunidad educativa.

- En el currículo se menciona que las unidades educativas deben acercar este diseño de planificaciones a la realidad de sus contextos, sin embargo, algunos centros educativos generan planificaciones "copiadas", pues la educación no se está pensando, se está repitiendo.

- Los formatos de planificaciones son referenciales, cada centro educativo puede diseñar su propio formato, ante esto, la mayoría de centros educativos no han generado sus propias planificaciones, ¿Cuáles son las razones?

- El ajuste curricular establece trabajar por destrezas, sin embargo, en los centros educativos se sigue trabajando por contenidos, es decir, repetir memorísticamente cada tema, sin desarrollar destrezas, habilidades ni competencias.

- En las aulas se visibiliza poco desarrollo del pensamiento racional y crítico, aspectos establecidos en el currículo.

- El currículo menciona interdisciplinaridad, mientras los centros educativos no saben cómo realizar esta afirmación. 
- El trabajo cooperativo es otra apuesta del currículo, sin embargo, algunos centros educativos no conocen cómo realizarlo y, por ende, se mantienen con el trabajo en grupos asumiendo que ello es cooperativo.

- La evaluación estudiantil en algunos centros es enfocada en la medición única de conocimientos, en definir lo malo y lo bueno, lo que sabe y no sabe el estudiante.

- El propósito de la evaluación es mejorar las prácticas educativas de los educadores, menciona el currículo, en los centros educativos pocos son los docentes que hacen reflexión de su práctica.

- La ley manifiesta evaluación integral, y me cuestiono la aplicación de las pruebas ser bachiller ¿son integrales?, las pruebas que aplica el Instituto Nacional de Evaluación al terminar cada subnivel se enfocan en ¿contenidos o destrezas? ¿cómo permite esa prueba cumplir con lo que se menciona en la ley, la evaluación no es únicamente una calificación?

Son muchas las realidades de los centros educativos, y otras mencionadas por los educadores con relación a los círculos de estudio impartido por los técnicos del MINEDUC y personalmente he presenciado una sobre las planificaciones, en esos espacios se dice que los $\mathrm{PCl}$ deben ser iguales en los centros educativos $i$ a caso no conocen el currículo? ¿o no conocen que cada centro debe contextualizar sus planificaciones y por ende ninguno debe ser igual?

Otros cuestionamientos que me surgen son con relación a: por qué si la Ley menciona que se debe incluir en los currículos de estudio, de manera progresiva, la enseñanza de, al menos, un idioma ancestral; el estudio sistemático de las realidades y saberes locales, la mayoría de los centros educativos no lo realizan, en las aulas poco se habla de las realidades locales, algunos de nuestros pueblos y comunidades indígenas reciben la educación en idiomas distintos a ellos. Nuestro Ecuador es diverso, por ello, la utilización de los libros deben ser solo una herramienta, no un elemento esencial, pues en los libros no se puede visualizar toda esa diversidad que existe en el país, además, no podemos seguir enseñando en la región amazónica únicamente matemáticas sueltas sin tomar en cuenta las consecuencias de la explotación petrolera 
¿dónde queda la contextualización, la interdisciplinaridad?

Con relación al derecho de recibir una educación actualizada ¿por qué los educadores siguen usando estrategias tradicionales, conocimientos obsoletos? ¿por qué no se incluyen las experiencias y los saberes ancestrales?

En la actualidad hay varios problemas como el abuso sexual, los homicidios, la delincuencia, violencia, etc., la presencia de estos problemas no son razones para cuestionarnos ¿qué estamos haciendo mal en la educación? ¿por qué hay tanta inconciencia humana? A todas estas inquietudes y realidades, que pueden estar supeditadas a distintas percepciones, ya que no hay verdades absolutas, surgen nuevas interrogantes: ¿por qué no se generan espacios de reflexión para tratar estos temas?, y si los hay, ¿dónde están los resultados? ¿con qué frecuencia se realiza? ¿cuáles son las acciones de mejora? Llevamos 2 años desde está actualización curricular y las problemáticas siguen visibles, muchos seguimos vendados ante las realidades concretas de cada lugar del país, pocos conocemos nuestros derechos.

En este sentido, el aporte de Jiménez (como se citó en Valenzuela, 2005) es interesante ya que, menciona: "Una de las contradicciones más grandes que vive la escuela son las tensiones entre la "currículo oficial" y la presencia del "currículo oculto", profundamente permeado por las culturas masivas" (p.353)

De ahí que, la implementación de este currículo es un reto, sobre todo para el mismo Ministerio de Educación, que ya ha dado grandes pasos al incluir estas medidas tanto en la LOEl como en su currículo. Entonces, para saber si existe la aplicación del currículo actual y mejor aún que exista innovaciones que respondan a nuestra realidad ecuatoriana, no es únicamente necesario realizar unas pruebas que midan conocimiento de alumnos, puesto que esto no garantiza transformación, ni tampoco es suficiente un cuestionario aplicado a docentes o una auditoría de aspectos administrativos, pues lo escrito en papel es muy hermoso y la aplicación sigue siendo incierta. Si realmente queremos transformar nuestra realidad, es imprescindible no solo aplicar una prueba de conocimientos a los estudiantes, sino escucharlos, permitir su participación "real" en la construcción de las planificaciones, de la toma 
de decisiones en su centro educativo, en todos los espacios. Además, es importante conocer los sentires de las familias, los sentires de las educadoras y los educadores, de los moradores de la comunidad, y a estos darles valor y construir juntos nuestras escuelas, con ellos y para ellos.

Así mismo, para sacar conclusiones de la implementación e innovación de las prácticas educativas partiendo desde el currículo, se hace necesaria la observación (no punitiva), visitas y acompañamiento oportuno y acogedor. Ya que, el enfoque en las cuestiones administrativas ha desviado la esencia de la educación y ha desgastado a nuestros educadores y educadoras. En este sentido, muchos de los centros educativos públicos, privados y fiscomisionales observados desde la experiencia educativa, "hacen actividades por cumplir" y mantienen prácticas educativas obsoletas, poco actualizadas, y nada contextualizadas; para ello puede existir varias razones.

\section{Posibles alternativas}

Hasta la actualidad los modelos educativos se han visto incapaces de dar respuesta a los grandes retos de la humanidad, entre ellos la sostenibilidad del medio ambiente, el cumplimiento de los derechos humanos y las problemáticas contextualizadas en el país, pues según la Fundación InteRed (2018).

Nos encontramos ante una crisis general del sistema socioeconómico y productivo (es decir, del modelo de desarrollo) que se evidencia por la crisis ambiental, la crisis de cuidados, la crisis de derechos humanos (políticos, sociales, culturales, económicos, ambientales, etc.) que generan exclusión y que afectan, aunque de diferente forma, al conjunto del planeta (p. 23)

Desde siempre muchos investigadores han apostado por la educación para lograr una transformación, en este sentido Lenin (citado en Valenzuela, 2005) comparte con certeza, que la educación y el estudio era la clave para avanzar hacia una sociedad sin clases sociales. Educar a la población en los principios de solidaridad y cooperación entre los pueblos y la defensa de sus raíces históricas, es la que debe ser fortalecida 
en los currículos escolares y en las acciones que se promueven desde la institución educativa.

Estos cambios en los centros educativos, así como en el currículo y la educación en general, parte de reestructurar el sistema educativo universitario, pues la preparación de estos profesionales son los que más tarde formarán a nuestros niños, niñas y adolescentes. Sin embargo, en la actualidad no poseen las herramientas necesarias para responder a la realidad actual. La existencia de un profesional de educación con vocación es muy poca, y las personas que están a cargo de nuestros niños, niñas y adolescentes, sobre todo en las parroquias, en la Amazonía y pueblos indígenas son bachilleres y con otra profesión distinta a educación.

Por ello, es necesario que desde el Ministerio de educación se realice formaciones integrales y permanentes a sus educadores $y$ educadoras, una formación que no únicamente se limite a enseñar como llenar formatos, o que las planificaciones sean estandarizadas como lo han llevado los distritos. Por tal razón, también es necesario repensar que formación estamos brindando a los responsables de los distritos, pues también pocas son las personas que conocen el currículo y que lo manejan.

La formación de nuestros educadores implica reaprender y aprender, para poder responder al contexto actual con una nueva era de estudiantes, y para que esto tenga su resultado eficaz hay que empezar viviendo la realidad y conociendo el currículo (A través de comunidades de aprendizaje), cada docente debe sentir y conectarse con la realidad, para que forme a sus estudiantes con el perfil de justos, innovadores y solidarios, ellos deben vivirlo. Que a partir del ejemplo se fortalecen estos procesos educativos, ya que, "no se puede educar o enseñar la democracia, desde prácticas y relaciones antidemocráticas; los valores deben ser vividos, no solo declarados o memorizados" (Valenzuela, 2005).

De ahí que, es necesario una formación a partir de la pedagogía del cuidado, con la finalidad de sanar heridas, eliminar rencores, liberar el alma y conectarse con la realidad. En este sentido la fundación InteRed (2018) nos aporta: 
La toma de conciencia de la necesidad de cuidados que tiene la vida (la nuestra y la del planeta) para poder existir, lleva inmediatamente a reconocerles un valor nuclear en nuestra existencia humana y debería llevar a ponerlos en un lugar preferente a la hora de tomar decisiones, políticas, educativas y de todo tipo (p. 26)

Cuidar a nuestros educadores y educadoras que tienen vocación y pasión por la educación, que buscan la transformación y luchan por las personas menos favorables.

A estas formaciones, es necesario sumarles las pedagógicas, brindándoles herramientas y estrategias que permitan generar espacios de participación de nuestros estudiantes, y esta es otra de las alternativas, propiciar real participación de adolescentes y jóvenes. Puesto que, al realizar los ajustes curriculares, únicamente se mencionó lo que se pretende, algunas estrategias en general, se creó instructivos, pero se les olvidó realizar formaciones con estrategias que permitan aplicar el currículo en el aula.

Finalmente, considero que se deben generar espacios de reflexión y diálogo, no se si estipulados a través de horas pedagógicas o algunos otros acuerdos, en la que nuestros estudiantes hagan vida su derecho de participación, pues como dice la siguiente frase:

"Si los responsables del mundo son todos venerablemente adultos, y el mundo está como está, ¿no será que debemos prestar más atención a los jóvenes?"

Benedetti (como se citó en Valenzuela, 2005)

Las palabras de Mario Benedetti son claras, y no quiere decir que debemos llegar disputas entre educadores y estudiantes, sino a mejorar nuestro diálogo, nuestro trabajo conjunto y cooperativo, pues en este proceso educativo educador/a y estudiantes aprendemos juntos. 


\section{Conclusiones}

Con los cambios actuales, los avances tecnológicos y el desarrollo social de la humanidad, se necesitan de sujetos abiertos a nuevas formas de pensar y de hacer las cosas; capaces de entender otros mundos culturales, otras formas de organizarse, otros puntos de vista, de escuchar, de respetar, tolerar, con capacidad para evaluar situaciones y tomar decisiones con autonomía. Aspectos que deben formarse desde un sistema educativo contextualizado, que respondan las problemáticas actuales, las necesidades de nuestros estudiantes y que no sean "currículos ocultos" que quieran generar más opresión y sumisión, por ello, debe partir de una formación humana flexible, creativa, reflexiva, crítica, y esto no se recibe pasivamente de nadie, sino que se autoconstruye a partir de múltiples experiencias de aprendizaje que partan de los intereses de los sujetos en formación, y su propio contexto cultural.

Finalmente, es necesario detenerse y hacer memoria, analizar y reflexionar, cómo está estructurado el currículo actual, cómo está su aplicación, qué mejoras hay que hacer, entre otros. De ahí que, es un reto para quienes soñamos con un mundo mejor lograr la transformación de estas realidades.

\section{Bibliografía:}

Casanova, M. A. (2006). Diseño curricular e innovación educativa. Madrid: La Muralla.

Fundación InteRed, (2018). Pedagogía de los cuidados-Aportes para su construcción. Recuperado de https://intered.org/ pedagogiadeloscuidados/wp-content/uploads/2018/05/MarcoTeorico Completo.pdf

GK (2018). La desigualdad es un problema grave (que los fanáticos económicos se nieguen a verlo es otra cosa). Recuperado de https://gk.city/2018/01/14/desigualdad-en-america-latina/

Ley Orgánica de Educación Intercultural (LOEI) Registro Oficial No. 417 de 31 de marzo de 2011, actualizado al 19 de mayo del 2017

Ministerio de Educación, (2016). Currículo de los niveles de educación Obligatoria. Ecuador. 
Ministerio de Educación (2017). Instructivo para la aplicación de la evaluación estudiantil. Quito, Ecuador.

Ministerio de Educación (2017). Instructivo para planificaciones curriculares para el sistema nacional de educación. Quito, Ecuador.

Rojas, C. (2012). Participación de los y las docentes en la transformación curricular. Revista electrónica Actualidades Investigativas en Educación, volumen (12), pp. 1-18.

REICE (2012). El diseño curricular como factor de calidad Educativa. Revista Iberoamerciana sobre calidad, eficacia y cambio en educación. Recuperado de http://www.redalyc.org/ pdf/551/55124841002.pdf

Valenzuela, J. (2005). El futuro ya fue. Juventud, educación y cultura. Buenos Aires, Argentina. 